\title{
Simplicity of Randomized, Controlled Trials of Percutaneous VERTEBROPLASTY
}

The readers of Pain Physician have been very fortunate to have the opportunity to read a very thorough review article on percutaneous procedures for the treatment of painful compression fractures. By and large, the authors have effectively discussed advantages and disadvantages of both percutaneous vertebroplasty and percutaneous kyphoplasty. The purpose of this invited commentary is to discuss one aspect of the paper; the ability to perform a randomized, controlled study of percutaneous vertebroplasty. There is little doubt that percutaneous kyphoplasty could be approached in the same fashion and equally little doubt that it will in the relatively near future.

Vertebroplasty is a well-established procedure that has been "seemed" efficacious in clinical practice extending over 10 years. Published case series examining thousands of patient cases attest to its "anecdotal" safety and efficacy.

However, the procedure is done as an off-label use of products approved by the FDA for other procedures. The call for randomized, clinical trials to prove the efficacy and safety of specific products for use in vertebroplasty has been the topic of much debate over the past few years. For example, Jarvik and Deyo wrote a letter to the editor in the American Journal of Neuroradiology calling for such a trial to clear the final, formal, scientific hurdle for a procedure that had become a standard of care for pain caused by vertebral compression fractures (1).

In their current article, Burton and Mendel reference several of the key papers calling for carefully monitored clinical trials; however, they assert that difficulty in enrolling patients and maintaining blindness to the control group will make randomized controlled trials for short and long-term outcomes of vertebroplasty problematic (2).

The authors of this commentary respectfully disagree with several aspects of this assertion, and offer the following references in the spirit of advancing the procedure for the benefit of the patient population now and in the future.
Over the past several years, there has been an increasing prevalence of placebo controlled studies for surgical procedures and non-surgical interventions. The success of these studies proves the efficacy of the study method itself in this arena; the inclusion of a control group is the basic premise of "good science".

More specifically, in 2002, Kallmes et al, demonstrated the feasibility of patient enrollment into a sham-controlled trial of verterboplasty(3). In that study, patients agreed to be enrolled knowing they might be randomized to the sham. The outcome of that study provided surprising insights, including the importance of the placebo effect as a valid study point in pain management and pain interventions.

Do et al have further demonstrated the feasibility with their prospective randomized study comparing vertebroplasty to medical therapy for acute vertebral compression fractures in 31 patients(4). Their findings indicate that all patients randomized to vertebroplasty had significant improvement in measured outcomes following vertebroplasty. Patients who were randomized to medical therapy had no improvement in pain, mobility, and narcotics intake. Additionally, all patients who were offered vertebroplasty after failure of medical therapy experienced significant improvement after their procedure. This is a strong finding for vertebroplasty as a pain intervention; it is more important as an indicator of the ability to enroll patients in a randomized, controlled trial involving non-intervention as a method of treatment.

Simultaneous with the presentation of papers by Kallmes et al and Do et al, was the announcement by Parallax Medical, Inc., of the first FDA approval of a randomized clinical trial of vertebroplasty products. (5) The trial design was approved by the FDA to include multiple centers, with a single-masked, randomized method including a placebo - a sham procedure. Patients who enroll in the trial and believe they have been randomized to the control group have the option of crossing over for vertebroplasty after a certain period, defined so patients would not be denied treatment for an extensive period. The Parallax trial has been underway for several months, involving many of the most prominent vertebroplasty practitioners at leading centers in the U.S. Patients have been enrolled and the study is proceeding according to the FDA-approved plan. Preliminary findings of the trial are expected to be reported later this year.

The authors of this commentary believe that the reported case series in the literature are supportive evidence, but that randomized controlled trials to show the efficacy and safety of the procedure according to FDA guidelines are appropriate. Not only is such a method of study appropriate, it has begun. Leading practitioners are choosing to participate in the trial, and patients are enrolling in it largely because of the design of the study and the ability to opt for crossover after an acceptable brief period of time following the initial treatment. The importance of the findings cannot be understated, and the importance of the trial should not be overlooked, both for the impact on vertebroplasty as a treatment for vertebral compression fractures, and influence on future trials of other interventions and surgical procedures.

\section{RefERENCES}

1. Jarvik, JG, Deyo, RA. Cementing the evidence: time for a randomized trial of vertebroplasty. AJNR 2000; 21:1373-1374

2. Burton AW, Mendel E. Vertebroplasty and kyphoplasty: a review. Pain Physician 2003;

3. Kallmes DF, Jensen ME, Marx WF, et al. A pilot study for a sham-controlled, randomized, prospective, crossover trial of percu taneous vertebroplasty, RSNA 2002.

4. Do HM, Marcellus ML, Weir RU, et al. Percutaneous vertebroplasty versus medica therapy for treatment of acute vertebral body compression fractures: a prospec tive randomized study. RSNA, 2002.

5. Parallax Medical, Inc., press release: FDA Approves Randomized Clinical Trial of Vertebroplasty Products by Parallax Medical, Inc., December 3, 2002, Chicago, IL. 
Joshua A. Hirsch, MD

Director of Interventional Neuroradiology

/ Endovascular Neurosurgery,

Assistant Professor of Radiology at Havard Medical School.

Massachusetts General Hospital, Gray 289,

Boston, MA 02214

E-mail: jhirsch@partners.org

\section{Huy M. Do, MD}

Interventional Neuroradiology

300 Pasteur Dr.

Rm. S-047 MC: 5105

Stanford, CA 94305

E-mail: huymdo@stanford.edu
David Kallmes, MD

Associate Professor of Radiology;

Interventional Neuroradiologist

Mayo Clinic - Rochester, MN

E-mail: Kallmes.david@mayo.edu

Richard M. Ruedy

Vice-President of Global, Clinical \&

Regulatory Affairs

Parallax Medical

940 Disc Drive

Scotts Valley, CA 95066-4544

E-mail: ric_ruedy@parallaxmedical.com
Jeffrey G. Jarvik, MD, MPH

Associate Professor of Radiology and

Neurological Surgery

Department of Radiology

University of Washington

1959 N.E. Pacific Street

Seattle, WA 98195-7115 